\title{
NGHIÊN CÚU PHƯƠNG PHÁP PHÁT TRIỂN HẸ QUY CHIẾU TỌA ĐỘ KHÔNG GIAN QUỐC GIA VN2000-3D KHI XUẤT HIỆN CÁC ĐIỂM CƠ SỞ MỚI
}

\author{
LƯO'NG THANH THACH \\ Trường Đại học Tài nguyên và Môi trường Hà Nội
}

\section{Tóm tắt:}

Trong tương lai, khi Việt Nam xây dựng thành công Hệ quy chiếu tọa độ không gian quốc gia VN2000 - 3D (Hệ tọa độ động), sau một thời gian khai thác và sử dụng sẽ có nhu cầu phát triển, mở rộng mạng lưới trắc địa GNSS cơ sở (xây dựng thêm các điểm cơ sở mới bằng công nghệ GNSS). Lúc này, chúng ta hoàn toàn có thể sử dụng các trị đo cũ và các trị đo mói để bình sai lại toàn bộ mạng lưới. Tuy nhiên, cách làm này không lợi ích về mặt thời gian, kinh tế và không phù hợp với sự tiến bộ của lý thuyết xử lý số liệu mạng lưới trắc địa hiện đại.

Trong bài báo khoa học này, sẽ trình bày cơ sở lý thuyết và kết quả thực nghiệm của bài toán bình sai truy hồi với phép biến đổi xoay (thuật toán T) để cập nhật các trị đo GNSS theo cách tiếp cận hiện đại xử lý số liệu mạng lưới trắc địa mà không phải bình sai lại mạng Iưới GNSS đã có từ trước, đáp ứng yêu cầu hiện đại của hệ thống thông tin trắc địa quốc gia.

\section{1. Đặt vấn đề}

Việc xây dựng hệ thống thông tin trắc địa quốc gia đã được các nước phát triển ở Châu Âu, Bắc Mỹ và Canada... hoàn thành từ cuối thập niên 70 của thế kỷ $X X$ và đã công bố trên nhiều hội nghị quốc tế về cơ sở dữ liệu (CSDL) trắc địa (Hà Minh Hòa (2014)). Việc lựa chọn hệ quản trị CSDL phụ thuộc vào mô hình dữ liệu. Hiện nay đang tồn tại bốn mô hình dữ liệu: mô hình mạng, mô hình phân cấp, mô hình quan hệ và mô hình hướng đối tượng. Mỗi loại mô hình có cách truy cập dữ liệu khác nhau. Các báo cáo, tham luận tại các hội nghị trắc địa quốc tế đã khẳng định rằng đối với dữ liệu trắc địa, mô hình phân cấp là hiệu quả nhất.

Việc lựa chọn các loại dữ liệu để lưu giữ trong các CSDL cũng là vấn đề quan trọng. Trong tài liệu (Tsherning C.C. (1979)) đã thông báo về 18 CSDL trắc địa trên thế giới, trong đó có 10 CSDL chứa các tọa độ và độ cao bình sai của các điểm trắc địa; 4 CSDL chứa các kết quả đo đạc và các mô tả về các điểm trắc địa ( $\mathrm{Mỹ}$, Canada, Đức và Đan Mạch).

12 trong 18 hệ thống thông tin trắc địa nêu trên có chức năng tính toán bình sai các mạng lưới trắc địa. Mối quan hệ giữa hệ thống thông tin trắc địa và các hệ thống thông tin khác là mối quan tâm hàng đầu của các nước, theo đó khuynh hướng rõ rệt nhất là hệ thống thông tin trắc địa là thành phần quan trọng nhất của các hệ thống thông tin khác. Ví dụ ở Đức, Áo, Hà Lan, Thụy điển đã xây dựng CSDL trắc địa trong hệ thống thông tin đất đai (LIS - Land Information System).

Theo tài liệu (The Europeaan Data and Analysis Center of the UELN), CSDL của mạng lưới độ cao Châu Âu bao gồm các

\footnotetext{
Ngày nhận bài: 20/4/2017, ngày chuyển phản biện: 24/4/2017, ngày chấp nhận phản biện: 03/5/2017, ngày chấp nhận đăng: 05/5/2017
} 
nước như Áo, Bỉ, Bosnia, Bulgari, CH Séc, Đan Mạch, Estonia, Phần Lan, Pháp, Đức, Hungari, Italy, Lithuania, Hà Lan, $\mathrm{Na} U \mathrm{U}, \mathrm{Ba}$ Lan, Bồ Đào Nha, Slovenia, Tây Ban Nha, Thụy Điển, Thụy Sĩ đang lưu giữa 5127 trị đo (các chênh đại lượng địa thế năng) của 3654 mốc độ cao (1 điểm khởi tính), với lượng đo dư là 1474 trị đo.

Ở Việt Nam, việc nghiên cứu xây dựng hệ thống thông tin trắc địa đã được thực hiện trong các công trình khoa học (Hà Minh Hòa, Trần Thùy Dương và nnk (2001); Hà Minh Hòa (2012); Hà Minh Hòa, Nguyễn Thi Thanh Hương (2015)).

Do nhu cầu phát triển của kinh tế - xã hội, Hệ quy chiếu tọa độ không gian quốc gia VN2000-3D sau một thời gian hoạt động, sẽ có nhu cầu phát triển mở rộng. Lúc này, xuất hiện thêm các điểm cơ sở mới (ở đất liền, ngoài các đảo, quần đảo... thuộc lãnh thổ Việt Nam, hoặc ở các nước lân cận) được đo bằng công nghệ GNSS. Yêu cầu kỹ thuật được đặt ra để giải quyết bài toán bổ sung các điểm cơ sở mới vào mạng lưới đã có như sau (Hà Minh Hòa, Nguyễn Thị Thanh Hương (2015)): Không bình sai lại toàn bộ mạng lưới, mà chỉ hiệu chỉnh các kết quả bình sai đã có và đang được lưu giữ trong "CSDL Kết quả bình sai" khi có các biến động về các dũ liệu đo đạc trong quá trình giải quyết bài toán nêu trên. Chúng ta cũng cần thiết phải chỉ ra rằng trong quá trình giải quyết bài toán được nêu trên, hoàn toàn có thể bình sai lại toàn bộ mạng lưới GNSS với các dữ liệu đo đạc bị biến động. Tuy nhiên, cách làm này không hiệu quả về kinh tế, thời gian, không ứng dụng được các tiến bộ hiện đại của Lý thuyết hiệu chỉnh toán học các mạng lưới trắc địa được phát triển trong những thập niên gần đây và không phản ánh được tính hiệu quả của việc xây dựng hệ thống thông tin trắc địa quốc gia.

\section{Giải quyết vấn đề}

Trong tài liệu (Hà Minh Hòa (2013)) đã trình bày đầy đủ cơ sở lý thuyết của phương pháp bình sai truy hồi với thuật toán $T$ và trong công trình (Hà Minh Hòa, Nguyễn Thi Thanh Hương (2015)) đã sử dụng phương pháp này để hiệu chỉnh các tuyến độ cao mới được đo đạc bổ sung mà không phải bình sai lại toàn bộ mạng lưới đã có. Trong bài báo khoa học này, chúng ta tiếp tục nghiên cứu thuật toán T để xử lý mạng lưới GNSS trong trường hợp đưa bổ sung các trị đo mới.

Khi bổ sung các trị đo mới thường có hai dạng bài toán sau:

Dạng 1: Bổ sung trị đo không làm thay đổi ẩn số; số.

Dạng 2: Bổ sung trị đo làm thay đổi ẩn

Trường hợp bài toán dạng 1 được giải quyết rất đơn giản và không phải là mục tiêu của bài báo khoa học này. Mục tiêu chính của bài báo khoa học này là giải quyết bài toán dạng 2 nêu trên.

Giả sử một mạng lưới GNSS gồm $\mathrm{n}$ trị đo, $k$ ẩn số. Sau khi thực hiện bình sai riêng rẽ bằng phương pháp bình sai truy hồi với thuật toán $T$ như đã trình bày trong tài liệu (Hà Minh Hòa (2013)), chúng ta có các giá trị được lưu giữ gồm ma trận $T_{n}, Y_{n}$ và dạng toàn phương $\Phi_{n}[P V V]_{n}$. Mạng lưới này bổ sung thêm n' trị đo mới và k' ẩn số mới. Khi tiến hành bình sai mạng lưới cũ và mạng Iưới mới bổ sung đồng thời với nhau chúng ta thực hiện các bước sau:

Bước 1: Lập ma trận ban đầu cho quá trình bình sai truy hồi

$$
\bar{T}_{0}=\left[\begin{array}{ll}
T_{n}^{k c k k} & 0 \\
0 & 10^{-6} \cdot E_{k^{\prime} x k^{\prime}}
\end{array}\right]_{\bar{k} \times \bar{k}},
$$

và vectơ số hạng tự do được biến đổi ban đầu: 


$$
\bar{Y}_{0}=\left[\begin{array}{l}
Y_{n} \\
0
\end{array}\right]_{\bar{k} \times 1},
$$

ở đây $\bar{k}=k+k^{\prime}$.

Bước 2: Lập hệ phương trình số cải chính cho n' trị đo mới. Đối với trị đo mới thứ i ( $i=n+1, n+2, \ldots, n+n ')$ chúng ta có phương trình số cải chính ở dạng sau:

$$
v_{i}=a_{i} \cdot \delta X+l_{i}^{(0)}
$$

với trọng số trọng số $p_{i}$

Bước 3: Triển khai bình sai truy hồi với phép biến đổi xoay đối với trị đo mới thứ i

Từ các biểu thức (1), (2), (3) chúng ta lập ma trận phụ:

$$
B_{i}=\left[\begin{array}{cc}
\bar{T}_{0+i-1} & \bar{Y}_{0+i-1} \\
\sqrt{p_{i}} \cdot a_{i} & -\sqrt{p_{i}} \cdot \ell_{i}^{(0)}
\end{array}\right],
$$

ở đây $\bar{T}_{0+i-1}$ và $\bar{Y}_{0+i-1}$ là ma trận tam giác trên được biến đổi và vectơ số hạng tự do được biến đổi nhận được sau khi đưa vào tính toán truy hồi i - 1 trị đo mới đầu tiên.

Thực hiện phép biến đổi xoay lần lượt từ hàng đầu tiên cho tới hàng thứ trận (4), chúng ta sẽ nhận được ma trận phụ biến đổi có cấu trúc:

$$
\bar{B}_{n+n^{\prime}}=\left[\begin{array}{cc}
\bar{T}_{0+i} & \bar{Y}_{0+i} \\
0 & \sqrt{\Delta \Phi_{i}}
\end{array}\right] .
$$

Bằng cách như vậy, sau khi đưa vào tính toán truy hồi tất cả n' trị đo mới, chúng ta nhận được ma trận tam giác trên được biến đổi $\bar{T}_{0+n^{\prime}}$ và vectơ số hạng tự do được biến đổi $\bar{Y}_{0+n^{\prime}}$.

Nghiệm của bài toán là: $\delta X=\bar{T}_{0+n^{\prime}}^{-1} \cdot \bar{Y}_{0+n^{\prime}}$ Dạng toàn phương:

$$
[P V V]_{n+n^{\prime}}=[P V V]_{n}+\sum_{i=1}^{n^{\prime}} \Delta \Phi_{n+i}
$$

Đối với trường hợp trị đo thứ i là các thành phần của vectơ baseline $\Delta X, \Delta Y, \Delta Z$ trên một cạnh giữa hai điểm GNSS, các phương trình số cải chính dạng (3) được đưa về dạng các phương trình của các trị đo độc lập theo phương pháp được trình bày trong (Hà Minh Hòa (2014)).

\section{Thực nghiệm}

Bình sai mạng lưới GNSS có thể được thực hiện bằng phương pháp truyền thống hoặc theo phương pháp bình sai truy hồi với phép biến đổi xoay Givens (thuật toán T).Để chứng minh ưu điểm trong việc bổ sung trị đo làm thay đổi ẩn số (thêm điểm mới) của thuật toán T, trong phần thực nghiệm dưới đây sẽ tiến hành tính toán bình sai mạng lưới GNSS với các trường hợp sau:

- Trường hợp 1: Bình sai tổng thể cả mạng lưới;

- Trường hợp 2: Bình sai một phần của mạng lưới;

- Trường hợp 3: Bình sai bổ sung trị đo làm thay đổi ẩn số (xuất hiện thêm điểm mới);

Mạng lưới thực nghiệm là "Mạng lưới cơ sở của các điểm địa động lực Miền Bắc", thời gian đo GNSS trên mỗi điểm liên tục với 4 ca đo trong 04 ngày đêm từ ngày $11 / 11 / 2013$ đến hết ngày $14 / 11 / 2013$. Các dữ liệu đo GNSS được xử lý bằng phần mềm Bernese ver5.0 trong ITRF2008 tương ứng với ellipsoid WGS84 quốc tế. Trong thực nghiệm chỉ lấy ca đo $1(11 / 11 / 2013)$ để đưa vào tính toán. Mạng lưới bao gồm 11 điểm, trong đó có 01 điểm gốc khởi tính (bảng 1) và 10 điểm mới cần xác định tọa độ. Sơ đồ lưới được cho như hình 1 dưới đây. (Xem hình 1, bảng 1)

Kết quả xử lý cạnh và ma trận tương quan được cho trong bảng 2. (Xem bảng 2)

\subsection{Bình sai tổng thể mạng lưới}

Kết quả bình sai tổng thể mạng lưới 
được thống kê trong bảng 3. (Xem bảng 3)

- Sai số trung phương trọng số đơn vị:

Dạng toàn phương $\Phi=[P V V]=0.001$

$$
m_{0}= \pm \sqrt{\frac{[P V V]}{n-t}}= \pm \sqrt{\frac{0.001}{3.24-3 .(11-1)}}= \pm 0.005(\mathrm{~m})
$$

\subsection{Bình sai một phần của mạng lưới}

Trong nội dung này, tiến hành bình sai truy hồi một phần của mạng lưới trên, bao gồm 09 điểm trong đó 01 điểm gốc, 08 điểm mới cần xác định tọa độ. Kết quả bình sai được trình bày trong bảng 4 dưới đây:

(Xem bảng 4)

Đánh giá độ chính xác:

- Dạng toàn phương: $\Phi=[P V V]=0.0003$

- Sai số trung phương trọng số đơn vị:

$m_{0}= \pm \sqrt{\frac{[P V V]}{n-t}}= \pm \sqrt{\frac{0.0003}{3.18-3 .(9-1)}}= \pm 0.003(\mathrm{~m})$
3.3. Bình sai bổ sung trị đo làm thay đổi ẩn số

Sử dụng các kết quả bình sai của mạng lưới đã giải quyết trong mục 3.2 đã được lưu giữ, để tiến hành bình sai bổ sung các trị đo khi xuất hiệnthêm hai điểm mới C070, C075.

Các kết quả của bài toán này được trình bày trong bảng 5 dưới đây: (Xem bảng 5 )

\section{Đánh giá độ chính xác:}

- Dạng toàn phương:

$\Phi=[P V V]=0.0003+0.0002=0.0005$

- Sai số trung phương trọng số đơn vị:

$$
m_{0}= \pm \sqrt{\frac{[P V V]}{n-t}}= \pm \sqrt{\frac{0.0005}{3.24-3 .(11-1)}}= \pm 0.003(\mathrm{~m})
$$

Nhận xét: Căn cứ vào bảng thống kê tọa độ ở các bảng 3 và bảng 5 , thấy rằng tọa độ của hai điểm $\mathrm{C} 070$ và $\mathrm{C} 075$ trong cả hai trường hợp tính toán bình sai tổng thể mạng

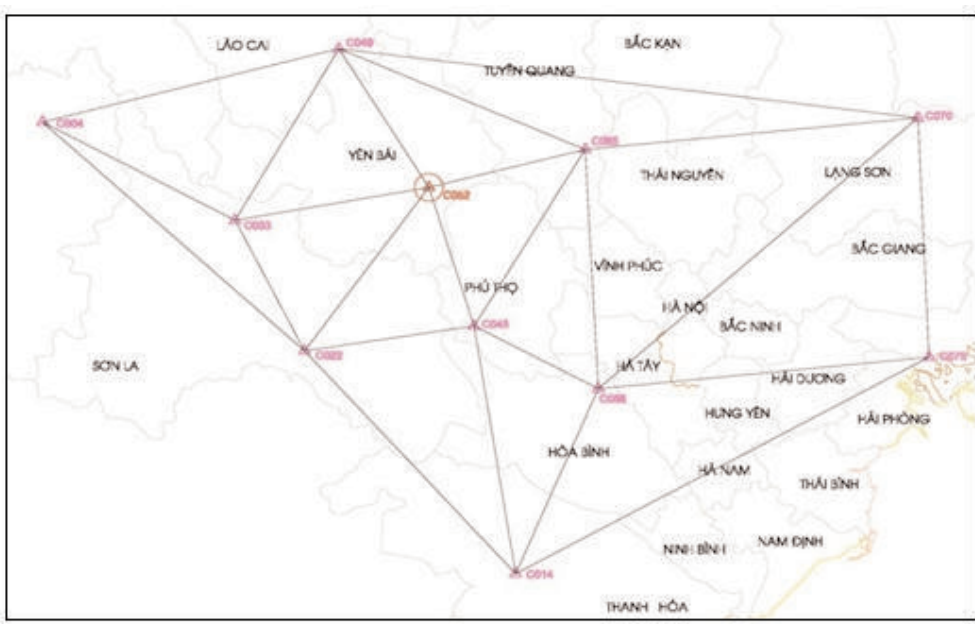

Hình 1: Mạng lưới cơ sở của các điểm địa động lực Miền Bắc

Bảng 1: Tọa độ gốc của mạng lưới (trong Hệ tọa độ vuông góc không gian)

\begin{tabular}{|c|c|c|c|c|}
\hline \multirow{2}{*}{} & \multirow{2}{*}{ Tên điểm } & \multicolumn{3}{|c|}{ Tọa độ không gian } \\
\cline { 3 - 5 } & & $\mathrm{X}(\mathrm{m})$ & $\mathrm{Y}(\mathrm{m})$ & $\mathrm{Z}(\mathrm{m})$ \\
\hline 1 & $\mathrm{C} 052$ & -1513906.489 & 5735082.184 & 2336981.806 \\
\hline
\end{tabular}


Bảng 2: Kết quả xử lý cạnh và ma trận tương quan

\begin{tabular}{|c|c|c|c|c|c|c|c|c|}
\hline \multirow{2}{*}{ Ngày đo } & \multirow{2}{*}{ Ca đo } & \multirow{2}{*}{ Cạnh đo } & \multicolumn{3}{|c|}{ Các thành phần của Baseline } & \multirow{2}{*}{\multicolumn{3}{|c|}{ Ma trận tương quan }} \\
\hline & & & $\Delta \mathrm{X}(\mathrm{m})$ & $\Delta \mathrm{Y}(\mathrm{m})$ & $\Delta Z(\mathrm{~m})$ & & & \\
\hline \multirow{3}{*}{$11 / 11 / 2013$} & \multirow{3}{*}{1} & \multirow{3}{*}{$\begin{array}{c}\mathrm{S} 1 \\
\mathrm{C} 052-\mathrm{C} 022\end{array}$} & 41534.9880 & 36369.4536 & -62460.0469 & 4.44E-05 & $-4.55 \mathrm{E}-05$ & $-2.28 \mathrm{E}-05$ \\
\hline & & & & & & & $1.58 \mathrm{E}-04$ & $6.52 \mathrm{E}-05$ \\
\hline & & & & & & & & 5.46E-05 \\
\hline \multirow{3}{*}{$11 / 11 / 2013$} & \multirow{3}{*}{1} & \multirow{3}{*}{$\begin{array}{c}\mathrm{S} 2 \\
\mathrm{C} 052-\mathrm{C} 033\end{array}$} & 74459.3920 & 22961.1070 & -8834.4626 & 5.45E-05 & $-5.74 \mathrm{E}-05$ & $-2.89 \mathrm{E}-05$ \\
\hline & & & & & & & $2.86 \mathrm{E}-04$ & 1.44E-04 \\
\hline & & & & & & & & 1.13E-04 \\
\hline \multirow{3}{*}{$11 / 11 / 2013$} & \multirow{3}{*}{1} & \multirow{3}{*}{$\begin{array}{c}\text { S3 } \\
\text { C052-C045 }\end{array}$} & -24890.1112 & 15063.6295 & -53268.7736 & $5.68 \mathrm{E}-05$ & $-6.15 \mathrm{E}-05$ & $-3.13 \mathrm{E}-05$ \\
\hline & & & & & & & $2.52 \mathrm{E}-04$ & 1.04E-04 \\
\hline & & & & & & & & 8.29E-05 \\
\hline \multirow{3}{*}{$11 / 11 / 2013$} & \multirow{3}{*}{1} & \multirow{3}{*}{$\begin{array}{c}\text { S4 } \\
\text { C052-C049 }\end{array}$} & 40326.6039 & -14646.2327 & 60592.5273 & 7.19E-05 & $-5.91 \mathrm{E}-05$ & $-3.66 \mathrm{E}-05$ \\
\hline & & & & & & & $2.85 \mathrm{E}-04$ & 1.19E-04 \\
\hline & & & & & & & & 1.00E-04 \\
\hline \multirow{3}{*}{$11 / 11 / 2013$} & \multirow{3}{*}{1} & \multirow{3}{*}{$\begin{array}{c}\text { S5 } \\
\text { C052-C065 }\end{array}$} & -63166.9249 & -24481.5728 & 17982.8555 & $4.66 \mathrm{E}-05$ & $-4.95 \mathrm{E}-05$ & $-2.43 \mathrm{E}-05$ \\
\hline & & & & & & & 2.06E-04 & $8.62 \mathrm{E}-05$ \\
\hline & & & & & & & & 6.58E-05 \\
\hline \multirow{3}{*}{$11 / 11 / 2013$} & \multirow{3}{*}{1} & \multirow{3}{*}{$\begin{array}{c}\mathrm{S} 6 \\
\mathrm{C} 004-\mathrm{C} 022\end{array}$} & -116712.9521 & 8895.5360 & -92393.5032 & $3.40 \mathrm{E}-05$ & $-3.19 \mathrm{E}-05$ & $-1.32 \mathrm{E}-05$ \\
\hline & & & & & & & 1.39E-04 & 4.93E-05 \\
\hline & & & & & & & & $4.13 E-05$ \\
\hline \multirow{3}{*}{$11 / 11 / 2013$} & \multirow{3}{*}{1} & \multirow{3}{*}{$\begin{array}{c}\text { S7 } \\
\text { C004-C033 }\end{array}$} & -83788.5470 & -4512.8154 & -38767.9226 & 3.67E-05 & $-3.15 \mathrm{E}-05$ & $-1.24 \mathrm{E}-05$ \\
\hline & & & & & & & $2.12 \mathrm{E}-04$ & 9.63E-05 \\
\hline & & & & & & & & 7.76E-05 \\
\hline & & & -117921.3334 & -42120.1489 & 30659.0709 & 5.34E-05 & $-4.66 \mathrm{E}-05$ & $-2.36 \mathrm{E}-05$ \\
\hline $11 / 11 / 2013$ & 1 & S8 & & & & & $2.43 \mathrm{E}-04$ & 9.29E-05 \\
\hline & & & & & & & & 7.46E-05 \\
\hline & & & 91835.6371 & -11227.2913 & 91501.8057 & 1.33E-05 & $-1.40 \mathrm{E}-04$ & $-4.31 \mathrm{E}-05$ \\
\hline $11 / 11 / 2013$ & 1 & $\begin{array}{c}\text { S9 } \\
\mathrm{C}-\mathrm{C} 4-\mathrm{C} 022\end{array}$ & & & & & $5.15 \mathrm{E}-04$ & 1.40E-04 \\
\hline & & & & & & & & 1.05E-04 \\
\hline & & & 25410.5405 & -32533.1079 & 100693.0805 & 1.43E-05 & $-1.55 \mathrm{E}-04$ & $\begin{array}{l}-4.89 E-05 \\
\end{array}$ \\
\hline $11 / 11 / 2013$ & 1 & $\begin{array}{c}\text { S10 } \\
\text { co14-C045 }\end{array}$ & & & & & 5.92E-04 & 1.90E-04 \\
\hline & & & & & & & & $1.48 \mathrm{E}-04$ \\
\hline & & & -28768.2340 & -37591.0007 & 75924.9261 & $1.58 \mathrm{E}-05$ & $-1.83 \mathrm{E}-04$ & $-5.45 \mathrm{E}-05$ \\
\hline $11 / 11 / 2013$ & 1 & $\begin{array}{c}S 11 \\
\mathrm{c} 014-\mathrm{C} 056\end{array}$ & & & & & $9.11 \mathrm{E}-04$ & $3.08 \mathrm{E}-04$ \\
\hline & & & & & & & & 2.19E-04 \\
\hline & & & -159338.7626 & -79891.9016 & 87084.0665 & 1.28E-05 & $-1.17 \mathrm{E}-04$ & $-3.57 E-05$ \\
\hline $11 / 11 / 2013$ & 1 & $\begin{array}{c}\text { S12 } \\
\text { co14-C075 }\end{array}$ & & & & & 5.63E-04 & $1.45 \mathrm{E}-04$ \\
\hline & & & & & & & & 1.17E-04 \\
\hline
\end{tabular}




\begin{tabular}{|c|c|c|c|c|c|c|c|c|}
\hline \multirow{2}{*}{ Ngày đo } & \multirow{2}{*}{ Ca đo } & \multirow{2}{*}{ Cạnh đo } & \multicolumn{3}{|c|}{ Các thành phần của Baseline } & \multirow{2}{*}{\multicolumn{3}{|c|}{ Ma trận tương quan }} \\
\hline & & & $\Delta \mathrm{X}(\mathrm{m})$ & $\Delta \mathrm{Y}(\mathrm{m})$ & $\Delta \mathrm{Z}(\mathrm{m})$ & & & \\
\hline \multirow{3}{*}{$11 / 11 / 2013$} & \multirow{3}{*}{1} & \multirow{3}{*}{$\begin{array}{c}\mathrm{S} 13 \\
\mathrm{C} 022-\mathrm{C} 033\end{array}$} & 32924.4043 & -13408.3506 & 53625.5809 & 3.82E-05 & $-2.86 \mathrm{E}-05$ & $-1.24 \mathrm{E}-05$ \\
\hline & & & & & & & $2.11 \mathrm{E}-04$ & 9.64E-05 \\
\hline & & & & & & & & 7.74E-05 \\
\hline \multirow{3}{*}{$11 / 11 / 2013$} & \multirow{3}{*}{1} & \multirow{3}{*}{$\begin{array}{c}\mathrm{S} 14 \\
\mathrm{C} 022-\mathrm{C} 045\end{array}$} & -66425.0956 & -21305.8302 & 9191.2700 & 3.74E-05 & $-3.75 \mathrm{E}-05$ & $-1.56 \mathrm{E}-05$ \\
\hline & & & & & & & $1.92 \mathrm{E}-04$ & 7.40E-05 \\
\hline & & & & & & & & 5.79E-05 \\
\hline \multirow{3}{*}{$11 / 11 / 2013$} & \multirow{3}{*}{1} & \multirow{3}{*}{$\begin{array}{c}\mathrm{S} 15 \\
\text { C033-C049 }\end{array}$} & -34132.7849 & -37607.3283 & 69426.9956 & $6.26 \mathrm{E}-05$ & $-3.06 \mathrm{E}-05$ & $-1.63 \mathrm{E}-05$ \\
\hline & & & & & & & 3.61E-04 & 1.71E-04 \\
\hline & & & & & & & & 1.40E-04 \\
\hline \multirow{3}{*}{$11 / 11 / 2013$} & \multirow{3}{*}{1} & \multirow{3}{*}{$\begin{array}{c}\text { S16 } \\
\text { C045-C056 }\end{array}$} & -54178.7742 & -5057.8922 & -24768.1556 & 4.05E-05 & $-4.09 \mathrm{E}-05$ & 2.25E-04 \\
\hline & & & & & & & $-1.75 \mathrm{E}-05$ & 9.40E-05 \\
\hline & & & & & & & & 7.43E-05 \\
\hline \multirow{3}{*}{$11 / 11 / 2013$} & \multirow{3}{*}{1} & \multirow{3}{*}{$\begin{array}{c}\mathrm{S} 17 \\
\mathrm{C} 045-\mathrm{C} 065\end{array}$} & -38276.8172 & -39545.2028 & 71251.6304 & 4.09E-05 & $-4.00 \mathrm{E}-05$ & $-1.67 \mathrm{E}-05$ \\
\hline & & & & & & & 2.49E-04 & 9.35E-05 \\
\hline & & & & & & & & 6.99E-05 \\
\hline \multirow{3}{*}{$11 / 11 / 2013$} & \multirow{3}{*}{1} & \multirow{3}{*}{$\begin{array}{c}\text { S18 } \\
\text { C049-C065 }\end{array}$} & -103493.5316 & -9835.3434 & -42609.6729 & 5.09E-05 & $-3.88 E-05$ & $-1.96 \mathrm{E}-05$ \\
\hline & & & & & & & $2.51 \mathrm{E}-04$ & 9.86E-05 \\
\hline & & & & & & & & 7.93E-05 \\
\hline \multirow{3}{*}{$11 / 11 / 2013$} & \multirow{3}{*}{1} & \multirow{3}{*}{$\begin{array}{c}\text { S19 } \\
\text { C049-C070 }\end{array}$} & -236747.6469 & -53312.6489 & -30292.2709 & 4.73E-05 & $-3.72 \mathrm{E}-05$ & $-1.88 E-05$ \\
\hline & & & & & & & 2.04E-04 & 7.34E-05 \\
\hline & & & & & & & & 6.21E-05 \\
\hline & & & 15901.9589 & -34487.3012 & 96019.7883 & $3.78 \mathrm{E}-05$ & $-3.67 E-05$ & $-1.69 \mathrm{E}-05$ \\
\hline $11 / 11 / 2013$ & 1 & C056-C065 & & & & & 1.94E-04 & 8.66E-05 \\
\hline & & & & & & & & 7.24E-05 \\
\hline & & & -117352.1560 & -77964.6107 & 108337.1875 & 3.77E-05 & $-3.40 \mathrm{E}-05$ & $-1.39 E-05$ \\
\hline $11 / 11 / 2013$ & 1 & C056-C070 & & & & & $1.70 \mathrm{E}-04$ & 7.18E-05 \\
\hline & & & & & & & & 6.02E-05 \\
\hline & & & -130570.1560 & -42300.8879 & 11159.1484 & 3.93E-05 & $-3.21 \mathrm{E}-05$ & -1.17E-05 \\
\hline $11 / 11 / 2013$ & 1 & C056-C075 & & & & & $2.16 \mathrm{E}-04$ & 9.00E-05 \\
\hline & & & & & & & & 7.17E-05 \\
\hline & & & -133254.1140 & -43477.3109 & 12317.4010 & 3.31E-05 & $-3.09 E-05$ & $-1.28 \mathrm{E}-05$ \\
\hline $11 / 11 / 2013$ & 1 & C065-C070 & & & & & $1.52 \mathrm{E}-04$ & 5.70E-05 \\
\hline & & & & & & & & 4.47E-05 \\
\hline & & & -13218.3709 & 35663.7197 & -97178.0447 & $3.58 \mathrm{E}-05$ & $-3.11 \mathrm{E}-05$ & $-1.17 \mathrm{E}-05$ \\
\hline $11 / 11 / 2013$ & 1 & C070-C075 & & & & & 1.55E-04 & 4.80E-05 \\
\hline & & & & & & & & 3.88E-05 \\
\hline
\end{tabular}


Bảng 3: Nghiệm và tọa độ sau bình sai

\begin{tabular}{|c|c|c|c|c|c|}
\hline TT & Tên điểm & Tọa độ gần đúng (m) & Nghiệm $\delta X$ & $\begin{array}{c}\text { Tọa độ sau bình } \\
\text { sai }(m)\end{array}$ & $\begin{array}{c}\text { SSTP vị trí điểm } \\
(\mathrm{m})\end{array}$ \\
\hline & \multirow{3}{*}{ C052 } & -1513906.489 & 0.000 & -1513906.489 & \multirow{3}{*}{ \pm 0.000} \\
\hline & & 5735082.184 & 0.000 & 5735082.184 & \\
\hline & & 2336981.806 & 0.000 & 2336981.806 & \\
\hline & \multirow{3}{*}{ C004 } & -1355658.549 & -0.003 & -1355658.552 & \multirow{3}{*}{ \pm 0.015} \\
\hline & & 5762556.102 & 0.008 & 5762556.110 & \\
\hline & & 2366915.262 & 0.008 & 2366915.270 & \\
\hline & \multirow{3}{*}{ C033 } & -1439447.097 & -0.002 & -1439447.099 & \multirow{3}{*}{ \pm 0.015} \\
\hline & & 5758043.291 & 0.002 & 5758043.293 & \\
\hline & & 2328147.343 & 0.003 & 2328147.346 & \\
\hline & \multirow{3}{*}{ C049 } & -1473579.885 & 0.003 & -1473579.882 & \multirow{3}{*}{ \pm 0.013} \\
\hline & & 5720435.951 & 0.003 & 5720435.954 & \\
\hline & & 2397574.333 & 0.001 & 2397574.334 & \\
\hline & \multirow{3}{*}{ C065 } & -1577073.414 & 0.002 & -1577073.412 & \multirow{3}{*}{ \pm 0.013} \\
\hline & & 5710606.611 & -0.012 & 5710600.599 & \\
\hline & & 2354964.662 & -0.010 & 2354964.651 & \\
\hline & \multirow{3}{*}{ C070 } & -1710327.528 & 0.001 & -1710327.527 & \multirow{3}{*}{ \pm 0.015} \\
\hline & & 5667123.300 & 0.005 & 5667123.305 & \\
\hline & & 2367282.063 & 0.000 & 2367282.063 & \\
\hline & \multirow{3}{*}{ C045 } & -1538796.600 & 0.004 & -1538796.596 & \multirow{3}{*}{ \pm 0.013} \\
\hline & & 5750145.814 & -0.008 & 5750145.806 & \\
\hline & & 2283713.032 & -0.007 & 2283713.025 & \\
\hline & \multirow{3}{*}{ C022 } & -1472371.501 & -0.004 & -1472371.505 & \multirow{3}{*}{ \pm 0.012} \\
\hline & & 5771451.638 & 0.011 & 5771451.649 & \\
\hline & & 2274521.759 & 0.011 & 2274521.770 & \\
\hline & \multirow{3}{*}{ C014 } & -1564207.138 & 0.000 & -1564207.138 & \multirow{3}{*}{ \pm 0.019} \\
\hline & & 5782678.929 & -0.012 & 5782678.917 & \\
\hline & & 2183019.953 & -0.006 & 2183019.947 & \\
\hline & \multirow{3}{*}{ C056 } & -1592975.374 & 0.001 & -1592975.373 & \multirow{3}{*}{ \pm 0.015} \\
\hline & & 5745087.921 & -0.004 & 5745087.917 & \\
\hline & & 2258944.877 & -0.003 & 2258944.874 & \\
\hline & \multirow{3}{*}{ C075 } & -1723545.901 & 0.005 & -1723545.896 & \multirow{3}{*}{ \pm 0.016} \\
\hline & & 5702787.033 & -0.008 & 5702787.025 & \\
\hline & & 2270104.025 & -0.004 & 2270104.021 & \\
\hline
\end{tabular}


Bảng 4: Nghiệm và tọa độ sau bình sai

\begin{tabular}{|c|c|c|c|c|c|}
\hline TT & Tên điểm & Tọa độ gần đúng (m) & Nghiệm סX & $\begin{array}{l}\text { Tọa độ sau bình } \\
\text { sai }(\mathrm{m})\end{array}$ & $\begin{array}{l}\text { SSTP vị trí điểm } \\
(\mathrm{m})\end{array}$ \\
\hline & \multirow{3}{*}{ C052 } & -1513906.489 & 0.000 & -1513906.489 & \multirow{3}{*}{ \pm 0.000} \\
\hline & & 5735082.184 & 0.000 & 5735082.184 & \\
\hline & & 2336981.806 & 0.000 & 2336981.806 & \\
\hline & \multirow{3}{*}{$\mathrm{CO04}$} & -1355658.549 & -0.002 & -1355658.551 & \multirow{3}{*}{ \pm 0.009} \\
\hline & & 5762556.102 & 0.005 & 5762556.107 & \\
\hline & & 2366915.262 & 0.005 & 2366915.267 & \\
\hline & \multirow{3}{*}{$\mathrm{C} 033$} & -1439447.097 & -0.001 & -1439447.098 & \multirow{3}{*}{ \pm 0.009} \\
\hline & & 5758043.291 & 0.000 & 5758043.291 & \\
\hline & & 2328147.343 & 0.001 & 2328147.344 & \\
\hline & \multirow{3}{*}{ C049 } & -1473579.885 & 0.002 & -1473579.883 & \multirow{3}{*}{ \pm 0.009} \\
\hline & & 5720435.951 & 0.005 & 5720435.956 & \\
\hline & & 2397574.333 & 0.003 & 2397574.336 & \\
\hline & \multirow{3}{*}{ C065 } & -1577073.414 & 0.000 & -1577073.414 & \multirow{3}{*}{ \pm 0.009} \\
\hline & & 5710606.611 & -0.001 & 5710600.611 & \\
\hline & & 2354964.662 & -0.001 & 2354964.661 & \\
\hline & \multirow{3}{*}{ C045 } & -1538796.600 & 0.004 & -1538796.596 & \multirow{3}{*}{ \pm 0.008} \\
\hline & & 5750145.814 & -0.012 & 5750145.802 & \\
\hline & & 2283713.032 & -0.011 & 2283713.021 & \\
\hline & \multirow{3}{*}{ C022 } & -1472371.501 & -0.003 & -1472371.504 & \multirow{3}{*}{ \pm 0.008} \\
\hline & & 5771451.638 & 0.006 & 5771451.643 & \\
\hline & & 2274521.759 & 0.005 & 2274521.765 & \\
\hline & \multirow{3}{*}{ C014 } & -1564207.138 & 0.002 & -1564207.136 & \multirow{3}{*}{ \pm 0.013} \\
\hline & & 5782678.929 & -0.026 & 5782678.903 & \\
\hline & & 213019.953 & -0.018 & 2183019.935 & \\
\hline & \multirow{3}{*}{ C056 } & -1592975.374 & 0.004 & -1592975.371 & \multirow{3}{*}{ \pm 0.011} \\
\hline & & 5745087.921 & -0.014 & 5745087.908 & \\
\hline & & 2258944.877 & -0.010 & 2258944.867 & \\
\hline
\end{tabular}

lưới (mục 3.1) và tính toán bình sai mạng lưới khi phát sinh trị đo làm thay đổi ẩn số (mục 3.3) có giá trị lệch nhỏ (cõ̃ cm). Giá trị cụ thể độ lệch trong hai trường hợp nêu trên xem trong bảng 6 . Điều này khẳng định tính đúng đắn của thuật toán. (Xem bảng 6)

\section{Kết luận}

Từ cơ sở lý thuyết và những kết quả thực nghiệm nêu trên chúng ta thấy rằng, việc ứng dụng phương pháp bình sai truy hồi với thuật toán $T$ hoàn toàn có thể đáp ứng cho bài toán cập nhật trị đo mới trong mạng lưới GNSS quốc gia.

Mạng lưới trắc địa quốc gia, sau nhiều năm khai thác và sử dụng sẽ có nhiều mốc không còn khả năng sử dụng (bị mất, bị 
Bảng 5: Nghiệm và tọa độ sau bình sai của 02 điểm bổ sung

\begin{tabular}{|c|c|c|c|c|}
\hline Tên điểm & Tọa độ gần đúng (m) & Nghiệm $\delta X$ & $\begin{array}{l}\text { Tọa độ sau bình } \\
\text { sai }(\mathrm{m})\end{array}$ & $\begin{array}{c}\text { SSTP vị trí điểm } \\
(\mathrm{m})\end{array}$ \\
\hline \multirow{3}{*}{ C070 } & -1710327.528 & 0.000 & -1710327.528 & \multirow{3}{*}{ \pm 0.017} \\
\hline & 5667123.302 & -0.006 & 5667123.296 & \\
\hline & 2367282.061 & -0.005 & 2367282.055 & \\
\hline \multirow{3}{*}{ C075 } & -1723545.898 & 0.002 & -1723545.896 & \multirow{3}{*}{ \pm 0.019} \\
\hline & 5702787.011 & -0.001 & 5702787.009 & \\
\hline & 2270104.009 & 0.000 & 2270104.008 & \\
\hline
\end{tabular}

Bảng 6: So sánh tọa độ điểm tính bổ sung trong hai trường hợp

\begin{tabular}{|c|c|c|c|c|}
\hline \multirow{2}{*}{ Tên điểm } & Tọa độ & Trường hợp 1 & Trường hợp 3 & Trị tuyệt đối độ lệch \\
\hline \multirow{3}{*}{ C070 } & $\mathrm{x}$ & -1710327.527 & -1710327.528 & 0.001 \\
\cline { 2 - 5 } & $\mathrm{y}$ & 5667123.305 & 5667123.296 & 0.009 \\
\cline { 2 - 5 } & $\mathrm{z}$ & 2367282.063 & 2367282.055 & 0.008 \\
\hline \multirow{3}{*}{ C075 } & $\mathrm{x}$ & -1723545.896 & -1723545.896 & 0.000 \\
\cline { 2 - 5 } & $\mathrm{y}$ & 5702787.025 & 5702787.009 & 0.016 \\
\cline { 2 - 5 } & $\mathrm{z}$ & 2270104.021 & 2270104.008 & 0.013 \\
\hline
\end{tabular}

chuyển dịch) do các tác động tự nhiên và nhân sinh. Việc loại bỏ các mốc không còn giá trị sử dụng này ra khỏi mạng lưới trắc địa quốc gia sẽ được tiếp tục nghiên cứu giải quyết trong các công trình tiếp theo.

Các thuật toán bình sai nêu trên sẽ góp phần hoàn thiện quá trình xây dựng cơ sở dữ liệu hệ thống thông tin trắc địa quốc gia, cho phép quản lý, khai thác và cập nhật các điểm mốc trắc địa quốc gia một cách khoa học và kịp thời. $\bigcirc$

\section{Tài liệu tham khảo}

[1]. Bùi Đăng Quang (2010). Hiệu chỉnh các trị đo trong mạng lưới độ cao nhà nước phục vụ xây dựng, cập nhật hệ thống thông tin trắc địa quốc gia. Tạp chí Khoa học Đo đạc và Bản đồ. Số 04 - tháng 06/2010, trg
$14-22$.

[2]. Hà Minh Hòa (1992). Lại một lần nữa về bình sai truy hồi các trị đo phụ thuộc. IZV. VUZOV. Geodezia I Aerophotoxemka, N2, c. 37 - 47. 1992. (Tiếng Nga).

[3]. Hà Minh Hòa (1996). Phương pháp bình sai truy hồi trong quy trình biến đổi xoay Givens. Báo cáo khoa học tại hội nghị khoa học lần thứ 12, trường Đại học Mỏ Địa chất, quyển 7, 1996, trg 73 - 77.

[4]. Hà Minh Hòa, Trần Thùy Dương và nnk (2001). Báo cáo khoa học và công nghệ của đề tài "Xây dựng cơ sở dữ liệu địa chính (trắc địa, hồ sơ địa chính) cấp tỉnh thuộc chương trình khoa học và công nghệ "Xây dựng một số giải pháp khoa học và công nghệ hỗ trợ công tác địa chính cấp tỉnh 
(thực nghiệm tại tỉnh Hà Nam)" cấp Tổng cục Địa chính giai đoạn 1999 - 2001", Hà Nội - 2001.

[5]. Hà Minh Hòa (2012). Nghiên cứu cơ sở khoa học của việc hoàn thiện hệ độ cao gắn liền với việc xây dựng hệ tọa độ động lực quốc gia. Báo cáo tổng kết khoa học và kỹ thuật của đề tài nghiên cứu khoa học cấp Bộ. Bộ Tài nguyên và Môi trường, Hà Nội 12/2012.

[6]. Hà Minh Hòa (2013). Phương pháp bình sai truy hồi với phép biến đổi xoay. NXB Khoa học và Kỹ thuật, 287 trg. Hà Nội 2013.

[7]. Hà Minh Hòa (2014). Phương pháp xử lý toán học các mạng lưới trắc địa Quốc gia. NXB Khoa học và Kỹ thuật, 244 trg, Hà
Nội 2014.

[8]. Hà Minh Hòa, Nguyễn Thị Thanh Hương (2015). Nghiên cứu hoàn thiện phương pháp xử lý toán học mạng lưới độ cao hạng I, II quốc gia trong hệ độ cao hiện đại ở Việt Nam. Báo cáo tổng kết khoa học và kỹ thuật. Đề tài cấp Bộ Tài nguyên và Môi trường, Hà Nội - tháng 8/2015.

[9]. Tsherning C.C. (1979). Management of Geodetic Data Status and Prospects. Report prepared for XVII General Assembly of IUGG/IAG. Canberra, Autralia, December, 1979.

[10]. The Europeaan Data and Analysis Center of the UELN. Bumdesamt fr Kartographie und Geodsie. 2013.O

\section{Summary}

\section{Research methodology for development of national space coordinates reference systems VN2000-3D when appearring new basis points}

\section{Luong Thanh Thach}

\section{Hanoi University of Natural Resources and Environment}

In the future, when Vietnam builds successfully national space coordinate reference system VN2000-3D (Dynamic Coordinate System), after a period of using, there will be a need to develop and expand the baseline GNSS geodetic network (building more new base stations using GNSS technology). At this time, we can completely use old and new measurements to adjustment the entire network. However, this approach is not useful in terms of time, economics and not consistent with the advancement of theory of data processing of modern geodetic network.

This paper presents the theoretical and empirical results of the recurrent adjustment and rotational transformation (T- algorithm) to update GNSS measurements according to modern treatment approaches of geodetic network data without having to adjust existing GNSS network to meet the modern requirements of the national geodetic information system. $O$ 